\title{
Author Correction: Isolation and characterization of halotolerant phosphate-solubilizing microorganisms from saline soils
}

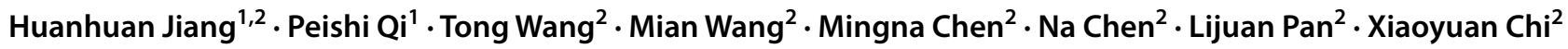

Published online: 8 January 2019

(c) King Abdulaziz City for Science and Technology 2019

\section{Author Correction: \\ 3 Biotech (2018) 8:461 \\ https://doi.org/10.1007/s13205-018-1485-7}

In the original article the acknowledgement section was incorrectly published. The correct acknowledgement is given below.

The State Key Laboratory of Urban Water Resource and Environment (2017DX15); The National Ten Thousand Youth Talents Plan of 2014 (W02070268), China Agriculture Research System (CARS-13 to XC), the National Natural Science Foundation of China (31701464), the Natural Science Fund of Shandong Province (ZR2017YL017), the Youth Scientific Research Foundation of Shandong Academy of Agricultural Sciences (2016YQN14), Agricultural Scientific and Technological Innovation Project of Shandong Academy of Agricultural Sciences (CXGC2016B02), Young Talents Training Program of Shandong Academy of Agricultural Sciences; the Basic Research Project of Qingdao (17-1-1-51-jch).

The original article can be found online at https://doi.org/10.1007/ s13205-018-1485-7.

Peishi Qi

qipeishi@163.com

$\triangle$ Xiaoyuan Chi

chi000@126.com

1 State Key Laboratory of Urban Water Resource and Environment (SKLUWRE), School of Environment, Harbin Institute of Technology, 150090 Harbin, China

2 Shandong Institute of Peanuts, 266100 Qingdao, China 